\title{
FY97 ICCS Prototype Specification
}

\author{
John Woodruff
}

$02 / 20 / 97$

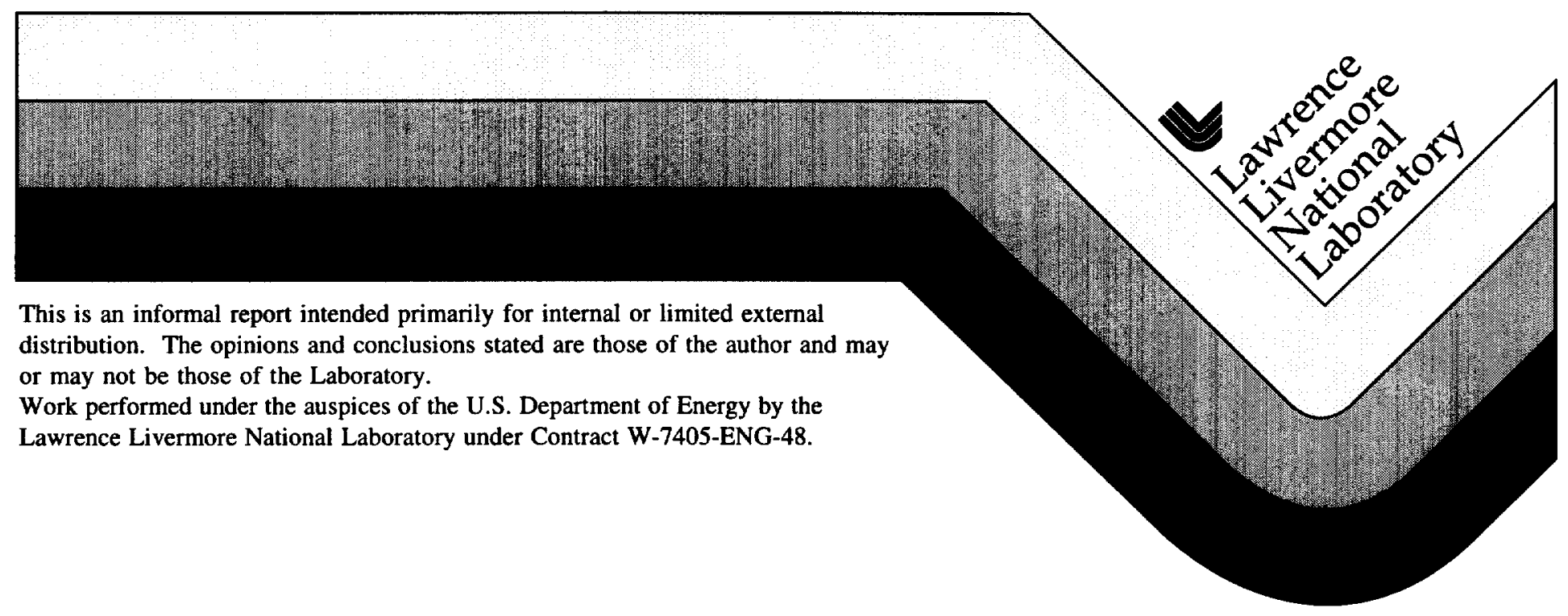




\section{DISCLAIMER}

This document was prepared as an account of work sponsored by an agency of the United States Government. Neither the United States Government nor the University of California nor any of their employees, makes any warranty, express or implied, or assumes any legal liability or responsibility for the accuracy, completeness, or usefulness of any information, apparatus, product, or process disclosed, or represents that its use would not infringe privately owned rights. Reference herein to any specific commercial product, process, or service by trade name, trademark, manufacturer, or otherwise, does not necessarily constitute or imply its endorsement, recommendation, or favoring by the United States Government or the University of California. The views and opinions of authors expressed herein do not necessarily state or reflect those of the United States Government or the University of California, and shall not be used for advertising or product endorsement purposes.

This report has been reproduced directly from the best available copy.

Available to DOE and DOE contractors from the Office of Scientific and Technical Information

P.O. Box 62, Oak Ridge, TN 37831

Prices available from (615) 576-8401, FTS 626-8401

Available to the public from the

National Technical Information Service

U.S. Department of Commerce

5285 Port Royal Rd.,

Springfield, VA 22161 


\title{
FY97 ICCS Prototype Specification
}

\author{
John Woodruff
}

20 Feb 97

\section{Introduction}

The ICCS software team will implement and test two iterations of their software product during FY97. The first of these iterations will concentrate on construction of selected framework components; the subsequent iteration will extend the product and perform measurements of performance based on emulated FEP devices. This document specifies the products to be delivered in that first prototype and projects the direction that the second prototype will take. Detailed specification of the later iteration will be written when the results of the first iteration are complete.

The selection of frameworks to be implemented early is made on a basis of risk analysis from the point of view of future development in the ICCS project. The prototype will address risks in integration of object-oriented components, in refining our development process, and in emulation testing for FEP devices. This document is a specification that identifies products and processes to undertake for resolving these risks.

The goals of this activity are to exercise our development process at a modest scale and to probe our architecture plan for fundamental limits and failure modes. The product of the iterations will be the framework software which will be useful in future ICCS code. Thus the FY97 products are intended for internal usage by the ICCS team and for demonstration to the FEP software developers of the strategy for integrating supervisory software with FEP computers. This will be the first of several expected iterations of the software development process and the performance measurements that ICCS will demonstrate, intended to support confidence in our ability to meet project RAM goals.

The design of the application software is being carried out in a separate WBS 1.5.2 activity. The design activity has as its FY97 product a series of Software Design Documents that will specify the functionality of the controls software of ICCS. During the testing of this year's prototypes, the application functionality needed for test will be provided by sample "maintenance" controls. These are early precursors of controls that can be used for low level device control. Since the devices under test will be represented by software emulation, these maintenance controls will be first drafts of the eventual product and will be useful only for ICCS team testing.

\section{Deliverable Products}

At two milestones in FY97, results of the ICCS prototype will be reported. Milestone 1 "Demonstrate first ICCS framework prototype" is scheduled for 
April 29 (the April prototype). Six framework software items will be delivered by the persons identified in the table. Remaining frameworks specified for ICCS in the Title I design are being deferred to future years.

Framework
Configuration
System Manager
Generic FEP
Status Monitor
Message Log
Sequence Control
History Archive
User Interface
Shot Setup
Shot Data Archive
Reservation

Responsible
Woodruff
Reynolds
Bettenhausen
Carey
Fong
Kettering
deferred
deferred
deferred
deferred
deferred

Section 4 of this document defines that functionality that will be delivered for each of the prototype frameworks in the April prototype. These code components will be tested together by executing several use cases that are listed in Section 6.1. These use cases are selected to demonstrate how the selected frameworks operate together.

April prototype components will be executed without widespread distribution in order to separate the concerns of functionality from the complex issue of CORBA distribution. A separate activity within the ICCS team is developing a capability for CORBA test generation that is independent of the framework prototype described here. Results of this testing will feed back into plans for later prototype iterations.

Milestone 2 "Demonstrate second ICCS framework prototype" is scheduled for September 26 (the Summer prototype). The second iteration will demonstrate CORBA distribution and will add selected functionality to be specified after first-round testing. A testing program that exercises the frameworks and measures the performance of network will be carried out during the second phase of the prototype. The product of the Summer prototype will be submitted for review and a report will be published that describes the test items, the process metrics gathered during development of both iterations, and the performance measurements.

The environment (processors, operating systems, network equipment) for the test is provided by WBS element 1.5.1. Work done in the April time frame will be hosted on the Sun workstations already installed and will run on the Solaris operating system. One VME-based Sparcstation belonging to the Alignment lab will be the host for the FEP testing. When ATM networks and PowerPC VME boards have been installed, and when compilers and CORBA middleware has been delivered for that environment, the software prototype 
will be ported. The activity of porting is expected to be straightforward because of our adoption of standard language and middleware.

\section{FEP Emulation}

It is premature to demand that NIF FEP software be capable of exercising a meaningful suite of device controls. Aside from the motor controllers available in the alignment laboratory, no physical device controls are scheduled for test early enough to participate in the April prototype. The first prototyping effort will instead be constructed by using software emulation. Control of devices will be emulated by constructing software that implements the interface required of FEP device objects.

GUI screens will be devised for individual device control and status report of each emulated device class. These GUI's are the first example of "maintenance" screens provided by ICCS.

The FEP software will be installed on a single platform (separate from the platform that runs the frameworks) and will communicate via CORBA. The April prototype will deploy on Solaris; subject to delivery of software tools, the Summer prototype should deploy on VxWorks.

\section{Framework Functionality in April Prototype}

ICCS software will use several multiple-use frameworks written in object-oriented Ada95. The prototype will construct representative parts of several frameworks and test the techniques for integration first by (conventional) linking into a single process and (subsequently in the Summer prototype) by CORBA distribution.

Each framework component has its requirements for the April prototype specified in this section. Each of these brief specifications is a subset of the required functionality documented in Software Requirement Specification documents. An indication of the likely extension to be produced in the Summer prototype follows for each.

\subsection{Configuration implements the following services:}

- Obtain device data from disk files.

- Accept control from SysMgr for FEP initialization.

- Invoke object initialization in the generic FEP.

- Create \& initialize device objects.

- Respond to success or failure reports from initialization.

- Provide object naming service for every client.

- In the Summer prototype, Oracle DB replaces disk file data.

\subsection{System Manager implements the following services:}

- Establish one running instance of ICCS. 
- Take user input (from a file) to specify "processing configuration"

- Provide data to other frameworks that specifies data filenames, initialization options, disposition of runtime records, etc.

- Report to (computer) operator the instance name of each running framework.

- In the Summer prototype, database tables are identified where appropriate instead of data filenames.

- In the Summer prototype, each framework is polled or dogwatched to monitor running process state

\subsection{Generic FEP prototype implements the following services:}

- Develop generic classes \& code which startup an FEP, startup and initialize objects that represent the I/O hardware devices, and startup and initialize NIF devices from the Configuration Framework

- Develop generic classes \& code for some common I/O devices such as binary $\mathrm{I} / \mathrm{O}$ and analog $\mathrm{I} / \mathrm{O}$

- Develop generic device emulators and deliver instances of a few representative emulators

- Develop a simple application which is based on the Generic FEP classes

- Modify CS\&T prototype to use the Generic FEP classes which apply

- In the Summer prototype, several FEP subsystems will be integrated with the frameworks and some hardware devices will be ready for test according to FEP project plans.

\subsection{Status Monitor implements the following services:}

- Establish multiple monitor objects for a single class of device within an emulated FEP. Monitor has settable frequency and tolerance.

- Instrument the framework for performance measures.

- Create one or two report receiver objects and dispatch to a GUI where status reports are displayed.

- Preliminary study of policies on dispatch aggregation (message per report; multiple report to supervisor fanout; multiple report to GUI fanout).

- In the Summer prototype the performance of different dispatch policies will be studied and reported.

\subsection{Message Log implements the following services:}

- Storage of free text messages with time-stamp and descriptor-set. 
- Retrieval of messages from specified time using descriptor-set algebra. Messages may be displayed on a terminal window.

- Retrieval of "current" log spools in real-time; retrieval of non-current entries does not spool.

- Resolve destination for stored log entries using information from system manager.

- In the Summer prototype, displays can be shown on a GUI.

- The Summer prototype adds configuration management of message text, including run-time parameterization.

\subsection{Sequence Control implements the following services:}

- Control constructs required to operate equipment implemented, except exceptions are not processed.

- Execution of hand-written sequences that dispatch to commands implemented in Ada.

- Prototype non-programmer's visual programming environment for sequences. Limited sequence correctness checking. A sequence developed under the interface cannot be executed in the April prototype.

- Summer prototype adds sequence code generation and the ability to execute a sequence developed with the non-programmer's visual programming environment.

\section{Software Development Process}

The team will exercise a preliminary version of the process specified by the NIF Ancillary Software QA Plan. The prototype process is based on structural modeling using Rose and construction of Ada code controlled by Apex. Experiments will be carried out to test the quality of Ada code automatically generated by Rose.

The April prototype will be compiled in a Gnat $\mathrm{RCI}$ view (owing to incomplete Apex product). Interface specifications will be peer reviewed and significant portions of delivered code will be walked through, unit tested and documented by the process prescribed by the SQA Plan.

Testing experience gained by executing the specified use-cases in April will be formalized during the Summer prototype. Definitions will be written for metric data, and the process for gathering development and testing data will be executed and reported during the Summer prototype. Initial prototypes of GUI screens will allow experiments in composing reusable graphic elements.

\section{Testing Strategy}

The testing that qualifies the April prototype will be based on the use cases described in the following paragraphs. Detailed scenarios for each use case 
will be generated in the Rose model and tests will be documented that define what units are tested and what results are observed.

Tests will be carried out using the "maintenance" GUI screens for direct observation, and using special purpose built test drivers for tests that require repetitive operation under computer control (as for example tests of average response time).

Measurements of performance on multiple computers can be performed by replicating the emulated devices and generating software-only signals to stress processor and network performance. Results of the April testing will let us judge the design approach from the point of view of the three risks (integration, process and emulation). The results will lead us to refine our Summer prototype plans.

\subsection{Use cases for execution in the April prototype:}

- System startup: using data from configuration files, start emulated devices on FEP, supervisory GUI(s), and message log. Details of the ICCS instance to be controlled by System Manager data files.

- Operator interacts with emulated devices using maintenance control panels.

- Execution is traced using the audit trail provided by Message Log.

- Device status is observed using Status Monitor updates on maintenance control panels.

- Policies for status update are varied using the Status Monitor configuration. Performance testing can be carried out by loading the FEP emulator with multiple (software) devices and monitoring message traffic and throughput.

- Hand-written control sequences will be constructed and executed to demonstrate how SCL capabilities will be deployed to application software engineers.

- Human interface studies will be performed to validate the SCL visual programming interface.

Testing during the Summer prototype phase will generate data of two kinds: process metric data and computer (and network) performance data.

Process metric data will be devised for the purpose of measuring reliability growth in order to satisfy NIF RAM requirements. The data gathering activity will be iterated during the prototyping activity.

Execution-time data will be collected that allow estimates to be calculated for required ICCS performance. Timing of network message delivery from emulated devices will allow us to extrapolate our ability to meet NIF performance requirements. Results from preliminary measurements carried 
out in the April prototype will permit the team to adapt the measures and to tune performance tradeoffs so as to assuage the risk of inadequate system performance. 


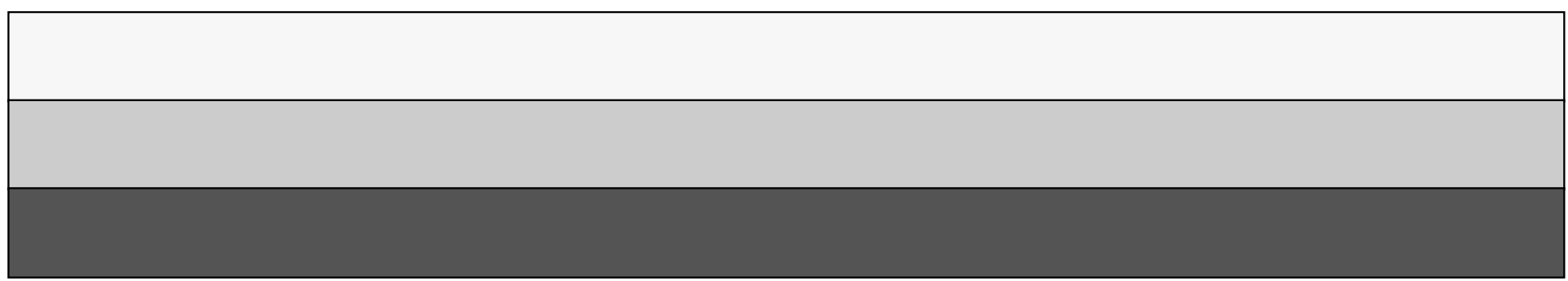

